\title{
Organic thin film photovoltaic cells based on planar and mixed heterojunctions between fullerene and a low bandgap oligothiophene
}

\author{
David Wynands, ${ }^{\text {a) }}$ Bert Männig, Moritz Riede, Karl Leo, Eduard Brier, Egon Reinold, and \\ Peter Bäuerle \\ Institut für Angewandte Photophysik, Technische Universität Dresden, 01062 Dresden, Germany \\ and Universität Ulm, 89069 Ulm, Germany
}

(Received 3 April 2009; accepted 14 July 2009; published online 11 September 2009)

\begin{abstract}
We present the material $\alpha$ - $\omega$-bis-(dicyanovinylen)-sexithiophen (DCV6T) as donor material in organic solar cells. A systematic study on the potential of DCV6T is given for different active layer concepts. DCV6T is a member of a class of acceptor-substituted oligothiophenes, which showed efficiencies of up to $3.4 \%$ and open circuit voltages $\left(V_{\mathrm{oc}}\right)$ of $1.0 \mathrm{~V}$, which were recently reported [K. Schulze et al. Adv. Mater. (Weinheim, Ger.) 18, 2875 (2006)]. To verify the potential of the material (DCV6T), organic solar cells with planar heterojunctions, bulk heterojunctions, and a hybrid-planar-mixed heterojunction are investigated. The planar heterojunction solar cells of DCV6T and C60 show the highest $V_{\text {oc }}$ of $0.90 \mathrm{~V}$. The mixed heterojunction solar cells have improved currents but a lower $V_{\text {oc }}$ of $0.82 \mathrm{~V}$. The solar cell using the hybrid-planar-mixed heterojunction achieves the best combination of parameters. It has a $V_{\text {oc }}$ of $0.88 \mathrm{~V}$, a short circuit current $\left(j_{\mathrm{sc}}\right)$ of $5.7 \pm 0.4 \mathrm{~mA} \mathrm{~cm}-2$, a fill factor of $41.6 \%$, and a power conversion efficiency of $2.1 \pm 0.2 \%$. () 2009 American Institute of Physics. [doi:10.1063/1.3204491]
\end{abstract}

\section{INTRODUCTION}

Organic solar cells have recently generated much interest for future electrical power generation due to their potentially low cost. They can be fabricated with a small amount of material at low temperatures and on flexible low-cost substrates. In 1986, Tang presented an organic solar cell reaching $1 \%$ efficiency ${ }^{1}$ which first introduced the concept of donor-acceptor (D-A) heterojunctions (HJs). They are crucial for efficient separation of the excitons created by photon absorption in the organic layer. This HJ can either be achieved by a planar structure, as it was the case in Tang's solar cell, or it can be realized in the form of an interpenetrating D-A network or so-called bulk or mixed HJ. ${ }^{2-5}$ Both types suffer from different limitations of the overall external quantum efficiency (EQE) given by

$$
\mathrm{EQE}=\eta_{a} \eta_{e d} \eta_{c t} \eta_{c c}
$$

where $\eta_{a}$ is the efficiency of absorption, $\eta_{e d}$ is the efficiency of exciton diffusion to a dissociating D-A interface, $\eta_{c t}$ is the probabilitiy that the charges are dissociated, and $\eta_{c c}$ is the efficiency of charge transport to the contacts. In planar HJ, only excitons that are created within the distance of the exciton diffusion length (several nanometers) from the D-A interface can be separated. Therefore, $\eta_{e d}$ is a limiting parameter for planar $\mathrm{HJ}$. In mixed $\mathrm{HJ}$, photogenerated excitons are always in proximity of a dissociating D-A interface and are therefore dissociated efficiently throughout the mixed film with $\eta_{e d}$ close to 1 . However, in particular, for small molecule materials, mixed layers are expected to have lower charge-carrier mobilities compared to layers of homogeneous composition and furthermore allow bimolecular charge recombination in the mixed layer, ${ }^{6}$ i.e., $\eta_{c c}$ is the main limiting

${ }^{a)}$ Electronic mail: david.wynands@iapp.de URL: http://www.iapp.de. parameter in mixed HJ. Therefore, it is a challenge to balance the thickness of the mixed HJ between the needs for high absorption (large thickness), on the one hand, and efficient charge transport (low thickness), on the other hand. This difficulty can be overcome by a hybrid planar-mixed molecular HJ (PM-HJ) consisting of a mixed layer of donor and acceptor molecules sandwiched between planar donor and acceptor layers. ${ }^{7-10}$ By using planar layer with thicknesses approximately equal to the respective exciton diffusion length, the PM-HJ has the high exciton diffusion efficiency of a mixed $\mathrm{HJ}$, but a better charge collection efficiency than a mixed $\mathrm{HJ}$ of the same overall thickness. Using the well known material system CuPc-C60, high efficiencies were reported with such a structure. ${ }^{7}$ The drawback of this material system is the low $V_{\text {oc }}$ of less than $0.6 \mathrm{~V}$, which is only about one-third of the optical gap energy. The reason for this is the low ionization potential (IP) of the donor $\mathrm{CuPc}$, which leads to significant energetic losses upon exciton separation.

In this paper, we present the material $\alpha$ - $\omega$-bis(dicyanovinylen)-sexithiophen (DCV6T) as the donor material in solar cells with C60 as the acceptor material. Due to the high IP of this material, high values for the $V_{\text {oc }}$ up to $0.9 \mathrm{~V}$ are achieved. To give a comprehensive view of the potential of this material we discuss all three different types of D-A HJ concepts, i.e., flat HJ devices, mixed HJ devices, and hybrid PM-HJ devices.

\section{EXPERIMENTAL}

The material discussed here (DCV6T) is an oligothiophene comprising of six $\alpha$-conjugated thiophene rings (6T) substituted with two terminal electron-withdrawing DCV groups, resulting in an A-D-A system concept recently introduced for analogous derivatives. ${ }^{11-13}$ It is one of the mem- 


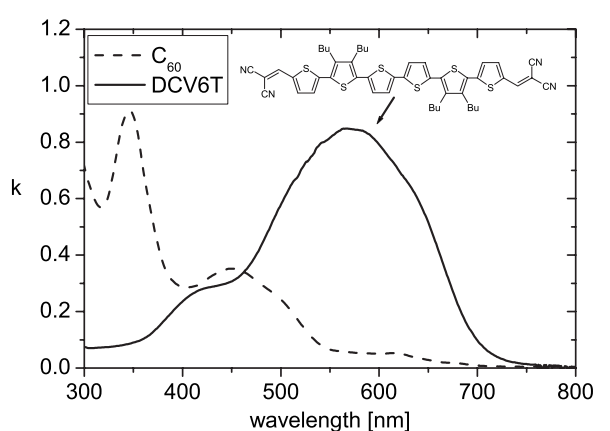

FIG. 1. Extinction coefficient spectra of DCV6T and C60. The inset shows the molecular structure of DCV6T.

bers of a homologous series of DCVnT that we are investigating. In a recent work, we demonstrated the effect of tuning of lowest unoccupied molecular orbital (LUMO) and highest occupied molecular orbital (HOMO) levels with varying length of the thiophene core of these molecules from $n=3$ to $n=6 .^{14}$ It was found that the LUMO energy remains almost constant with $n$, whereas the HOMO energy shifts to higher energies with every additional thiophene ring. The LUMO and HOMO energies of DCV6T determined by cyclic voltammetry $(\mathrm{CV})$ are -3.44 and $-5.24 \mathrm{eV}$, respectively. However, from ultraviolet photon spectroscopy (UPS) of thin films of DCV5T, it is known that the HOMO energy is underestimated in CV compared to UPS measurements. ${ }^{14}$ According to the observed variations with $n$, the IP value of DCV6T can be estimated to be approximately $0.1 \mathrm{eV}$ lower than that of DCV5T $(-5.6 \mathrm{eV})$. This corresponds well to measurements of $V_{\mathrm{oc}}$ in solar cells with DCVnT/C60 HJs in which DCV6T acts as good donor material in HJ with C60. ${ }^{14}$ It offers efficient exciton separation due to the offsets in the corresponding HOMO and LUMO energies and still high $V_{\mathrm{oc}}$ is achieved due to the high IP, as it is the case for DCV5T. ${ }^{1}$ The optical absorption spectrum of a DCV6T thin film is presented in Fig. 1. DCV6T thin films exhibit a maximum extinction coefficient at $580 \mathrm{~nm}$ of $\alpha=1.89 \times 10^{5} \mathrm{~cm}^{-1}$. Together with $\mathrm{C} 60$, the whole spectral region from 300 to $680 \mathrm{~nm}$ is covered.

The layer structures of the investigated solar cells are sketched in Fig. 2. In detail the structure is as follows: (A) ITO/Au (1)/p-NPB (15)/NPB (5)/DCV6T (8.8)/C60 (52)/ Bphen (6)/Al (100); (B) ITO/Au (1)/p-NPB (15)/NPB (5)/ DCV6T (11.7)/C60 (52)/Bphen (6)/Al (100); (C) ITO/Au (1)/p-NPB (15)/NPB (5)/DCV6T (17.6)/C60 (52)/Bphen (6)/Al (100); (D) ITO/Au (1)/p-DiNPB (30)/DiNPB (5)/ DCV6T (17.6)/C60 (52)/Bphen (6)/Al (100); (E) ITO/Au (1)/p-NPB (15)/NPB (5)/DCV6T:C60 (12.4)/C60 (32.5)/ Bphen (6)/Al (100) (F) ITO/Au (1)/p-NPB (15)/NPB (5)/ DCV6T:C60 (24.9)/C60 (32.5)/Bphen (6)/Al (100); (G) ITO/Au (1)/ $p$-NPB (15)/NPB (5)/DCV6T:C60 (37.7)/C60 (32.5)/Bphen (6)/Al (100); and (H) ITO/Au (1)/p-TNATA (30)/p-NPB (10)/NPB (5)/DCV6T (5.9)/DCV6T:C60 (31.4)/ C60 (32.5)/Bphen (6)/Al (100).

All thicknesses are given in nanometers. The mixing ratio of the BHJ layers in devices E-H is 1:2.2 DCV6T:C60. The gold interlayer on indium tin oxide (ITO) preserves Ohmic contact behavior under prolonged irradiation with UV
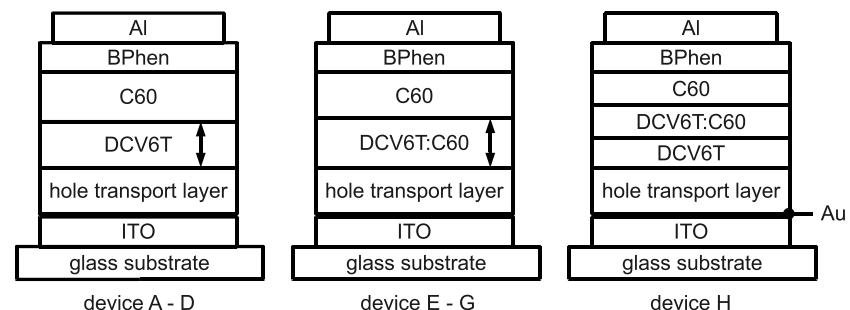

FIG. 2. Layer structure of the investigated devices. Devices A-D are flat HJs, devices E-G are mixed HJs and device $\mathrm{H}$ is a hybrid PM HJ. The arrows illustrate the thickness variation.

light. All devices use a pristine C60 layer at the electron contact side due to the good charge transport capability of C60. In mixed HJ devices, this pristine layer also contributes to photocurrent generation and therefore already acts like the $n$-side part of a full hybrid PM-HJ device. To complete a full hybrid PM-HJ device a pristine layer of the donor at the $p$-side is required in addition. The thickness of the pristine C60 layer is identical for the mixed HJ and hybrid PM-HJ devices which enables a direct comparison. The layer thicknesses for the hybrid PM-HJ are not varied but set to values that promised an optimized result considering the results from cells A-G. The characteristic parameters of the investigated solar cells can be seen in Table I.

All devices were prepared on semitransparent ITOcoated glass substrates (thin Film Devices, Inc., sheet resistance of $\leqslant 30 \Omega \mathrm{sq}^{-1}$ ) and had an average size of $3.0-7.5 \mathrm{~mm}^{2}$. The substrates were cleaned using aqueous detergent, acetone, and ethanol in ultrasonic bath. All organic layers were deposited on the substrates by thermal evaporation in an ultra high vacuum multichamber system with typical pressure of $10^{-7}$ mbar. The layer thicknesses were determined using a quartz crystal monitor that was calibrated by placing a second crystal at the sample position before. The thickness of the DCV6T layer was checked by measuring the height of a test layer using an atomic force microscope. $\quad N, N^{\prime}$-di(naphthalen-1-yl)- $N, N^{\prime}$-diphenyl-benzidine $\left(\alpha\right.$-NPD), $N, N^{\prime}$-Diphenyl- $N, N^{\prime}$-bis $\left(4^{\prime}\right.$ - $[N, N$-bis(naphth-1yl)-amino]-biphenyl-4-yl)-benzidine

(Di-NPD), and $4,4^{\prime}, 4^{\prime \prime}$-tris(1-naphthylphenylamino)-triphenylamine (TNATA) were used as hole transport layers (HTLs). For the

TABLE I. Open circuit voltage $V_{\mathrm{oc}}$ short circuit current density $J_{\text {sc }}$, fill factor FF, intensity I of simulated sunlight and power conversion efficiency $\eta_{\mathrm{PCE}}$ for the investigated solar cells A-H. The efficiency is not corrected to the spectral mismatch. Furthermore, $J_{\mathrm{sc}(100)}$ represents the linear interpolated value for $j_{\mathrm{sc}}$ at $100 \mathrm{~mW} \mathrm{~cm} \mathrm{~m}^{-2}$ simulated sunlight.

\begin{tabular}{lcccccc}
\hline \hline & $\begin{array}{c}V_{\mathrm{oc}} \\
\text { Device }\end{array}$ & $\begin{array}{c}J_{\mathrm{sc}} \\
(\mathrm{mA} \mathrm{cm})\end{array}$ & $\begin{array}{c}I \\
\left(\mathrm{~mW} \mathrm{~cm}^{-2}\right)\end{array}$ & $\begin{array}{c}J_{\mathrm{sc}(100)} \\
\left(\mathrm{mA} \mathrm{cm}^{-2}\right)\end{array}$ & $\mathrm{FF}$ & $\begin{array}{c}\eta_{\mathrm{PCE}} \\
(\%)\end{array}$ \\
\hline $\mathrm{A}$ & 0.90 & 7.7 & 117 & 6.6 & 0.531 & 3.1 \\
$\mathrm{~B}$ & 0.87 & 7.0 & 116 & 6.0 & 0.503 & 2.6 \\
$\mathrm{C}$ & 0.89 & 6.3 & 116 & 5.5 & 0.426 & 2.1 \\
$\mathrm{D}$ & 0.88 & 7.2 & 134 & 5.4 & 0.288 & 1.4 \\
$\mathrm{E}$ & 0.80 & 6.8 & 134 & 5.1 & 0.437 & 1.8 \\
$\mathrm{~F}$ & 0.82 & 8.8 & 128 & 6.9 & 0.403 & 2.3 \\
$\mathrm{G}$ & 0.81 & 9.6 & 135 & 7.1 & 0.381 & 2.2 \\
$\mathrm{H}$ & 0.88 & 10.6 & 128 & 8.2 & 0.416 & 3.0 \\
\hline \hline
\end{tabular}


a)

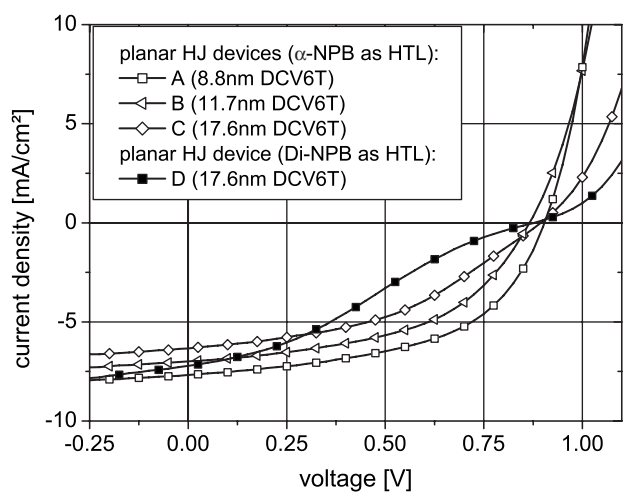

b)

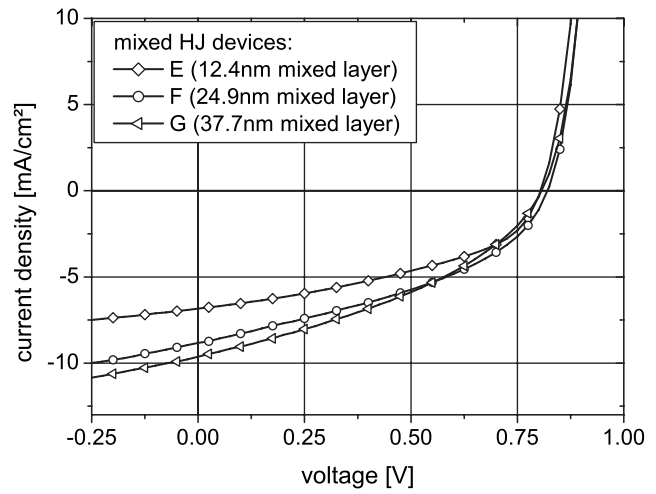

FIG. 3. (a) Current density vs voltage $(J-V)$ characteristics of planar HJ solar cells with DC6T/C60. The thickness of the DCV6T layer is varied from 8.8 to $17.6 \mathrm{~nm}$. For devices A-C, $\alpha$-NPB was used as hole transport material, while Di-NPB was used in the case of device D. (b) $J-V$ characteristics of mixed HJ solar cells with DCV6T:C60. The thickness of the mixed layer is varied from 12.4 to $37.7 \mathrm{~nm}$.

p-doping of the Di-NPB layer in device D 2-[4(dicyanomethylidene)-2,3,5,6-, tetrafluorocyclohexa-2,5dien-1-ylidene]propanedinitrile (F4TCNQ) was used with a ratio of $25 \mathrm{~mol} \%$. In devices $\mathrm{A}-\mathrm{C}$ and $\mathrm{E}-\mathrm{H}$ an acceptor dopant from Novaled AG (Dresden, Germany) was used with ratios of $15 \mathrm{~mol} \%$ in $\alpha$-NPD and $7 \mathrm{~mol} \%$ in TNATA. In all cases, the conductivity of the doped layers was above $10^{-6} \mathrm{~S} \mathrm{~cm}^{-1}$ at room temperature. Thus the voltage drop across the HTL can be neglected. Between the $p$-doped HTL and the DCV6T layer, a $5 \mathrm{~nm}$ undoped hole transport interlayer was inserted. C60 was used as electron transport material and 4,7-diphenyl-1,10-phenanthroline (BPhen) as exciton blocker and aluminium as cathode. The materials were cleaned at least twice before use by thermal gradient sublimation except for DCV6T and the dopant that were used as received. The $J-V$ characteristics were recorded with a source measure unit 236 SMU (Keithley) under a sun simulator (Hoenle $\mathrm{AG}$ ) with an average intensity of $115-135 \mathrm{~mW} \mathrm{~cm}^{-2}$, as determined by an outdoor reference cell (Fraunhofer Institute for Solar Energy Systems ISE, Freiburg, Germany). For the EQE measurement, a homemade setup based on a xenon arc lamp and a grating monochromator (Acton Research Corporation) was used. The setup was calibrated using a Newport power meter. All electrical measurements were performed under nitrogen atmosphere. Absorption spectra were recorded on quartz glass with a standard spectrometer from Shimadzu.

\section{RESULTS}

\section{A. Planar HJ devices}

Figure 3(a) shows the $J-V$ characteristics of the planar HJ cells (A, B, C, and D). All of them achieve high $V_{\mathrm{oc}}$ values of up to $0.9 \mathrm{~V}$. This is due to the high IP of DCV6T which results in a large interfacial energy gap $\Delta E_{\mathrm{AD}}$ between the DCV6T HOMO and the C60 LUMO. However, compared to the previously published material DCV5T, $V_{\text {oc }}$ here is about $0.1 \mathrm{eV}$ lower than in solar cells with a DCV5T/C60 planar HJ $\left(V_{\mathrm{oc}}=1.0 \mathrm{~V}\right){ }^{13}$ This difference is caused by the difference in the IP of the two oligothiophene derivatives. ${ }^{14}$ By adding one thiophene unit to the core of the oligothiophene derivatives, the IP shifts to higher energy, leading to a reduced energy difference between HOMO-donor and LUMO-C60. Since the $V_{\text {oc }}$ is known to linearly correlate with this difference, the estimate of the IP of DCV6T $(5.5 \mathrm{eV})$ being $0.1 \mathrm{eV}$ lower than that of DCV5T $(5.6 \mathrm{eV})$ is confirmed. $^{15-17}$

Figure 3(a) also shows that for thicker layers of DCV6T, in particular, for device $\mathrm{C}(17.6 \mathrm{~nm})$, the fill factor $(\mathrm{FF})$ is strongly reduced and signs of an $\mathrm{S}$ shape appear in the $I-V$ characteristic. Especially in device D, where Di-NPB is used as HTL, the $\mathrm{S}$ shape is very pronounced. The same behavior was already reported for DCV5T/C60 planar HJ solar cells. ${ }^{13}$ It is associated with a hole injection barrier between the HTL $(\alpha-\mathrm{NPB}$ or Di-NPB) and the donor material (DCV6T), resulting from the difference in HOMO energy. The HOMO energies of Di-NPB $(-5.4 \mathrm{eV})$ and $\alpha$-NPB $(-5.4 \mathrm{eV})$ lie higher than the HOMO energy of DCV6T. Therefore, hole injection to DCV6T is hindered. Analogously, holes lose free energy when they leave DCV6T to the HTL. This loss negatively affects the FF but does not change the static quantity of $V_{\text {oc }}$. It was shown by Uhrich et al. ${ }^{18}$ that in case of an injection barrier between HTL and donor the photocurrent is mainly driven by the concentration gradient when voltages near $V_{\mathrm{oc}}$ are applied. Therefore devices with thinner layers of DCV6T show a smaller decrease in FF than devices with thicker layers of DCV6T, where the charge carrier density gradient is not enough to maintain the photocurrent over the whole layer thickness and the charges recombine before they are collected.

Another important result is that $j_{\mathrm{sc}}$ decreases for increasing layer thickness of DCV6T. Device A shows the highest $j_{\mathrm{sc}}\left(6.6 \mathrm{~mA} \mathrm{~cm}^{-2}\right.$ at $\left.100 \mathrm{~mW} \mathrm{~cm}^{-2}\right)$ with a DCV6T layer thickness of $8.8 \mathrm{~nm}$. This is caused by the very small exciton diffusion length in DCV6T. From EQE measurements at certain wavelengths $(546,578$, and $625 \mathrm{~nm})$, the exciton diffusion length is estimated to be $L=9 \pm 3 \mathrm{~nm}$. Here we follow the approach of Yoo et al. ${ }^{19}$ Due to uncertainties in the handling of the exciton reflecting behavior at the DCV6T/ $\alpha$-NPB interface, this value cannot be calculated more accurately. Nevertheless, the result shows that the limit of $\eta_{e d}$ is already reached at low DCV6T layer thicknesses of about $10 \mathrm{~nm}$. This limitation can only be overcome by the mixed or the hybrid concept. 
a)

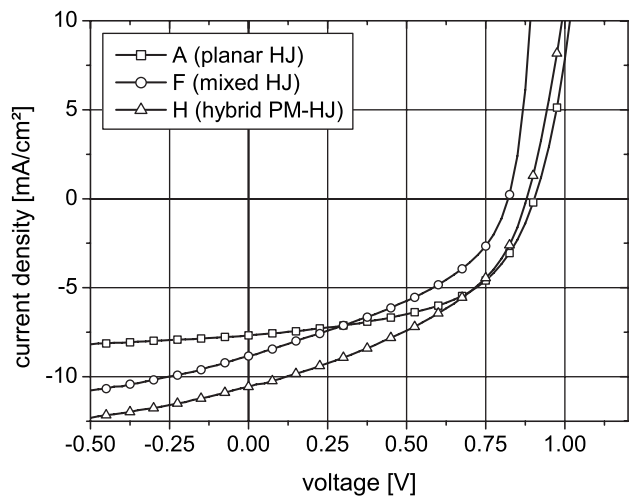

b)

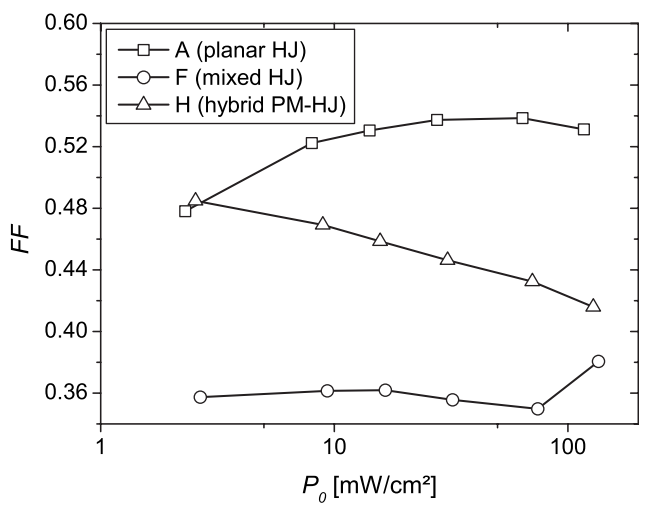

FIG. 4. (a) Current density vs voltage ( $J-V)$ characteristics of cell A (planar HJ, squares), cell F (mixed HJ, circles), and cell H (hybrid PM-HJ, triangles). (b) Development of the FF with intensity for cell A, cell F, and cell $\mathrm{H}$.

\section{B. Mixed HJ devices}

Figure 3(b) shows the $I-V$ characteristics of the mixed HJ solar cells (E, F, and G). For increasing mixed layer thickness, $j_{\mathrm{sc}}$ increases, whereas FF decreases, which is expected for the mixed HJ structure. ${ }^{20}$ While $\eta_{e d}$ can be assumed to be nearly unity, $\eta_{c c}$ limits the efficiency of mixed HJ solar cells. Since nearly every exciton will be dissociated into charge carriers, $j_{\mathrm{sc}}$ in mixed HJ solar cells is considerably higher than that in planar HJ solar cells. This can be seen at the mixed HJ device F, which contains an overall amount of $7.7 \mathrm{~nm}$ DCV6T and $49.6 \mathrm{~nm} \mathrm{C60} \mathrm{and} \mathrm{shows} \mathrm{a}$ higher $j_{\mathrm{sc}}\left(6.9 \mathrm{~mA} \mathrm{~cm}^{-2}\right.$ at $\left.100 \mathrm{~mW} \mathrm{~cm}^{-2}\right)$ than the planar $\mathrm{HJ}$ device $\mathrm{A}\left(6.6 \mathrm{~mA} \mathrm{~cm}^{-2}\right.$ at $\left.100 \mathrm{~mW} \mathrm{~cm}^{-2}\right)$, which contains a comparable amount of $8.8 \mathrm{~nm}$ DCV6T and $52 \mathrm{~nm} \mathrm{C60} \mathrm{as}$ bilayer. However, this advantage is offset by the low FF of device $\mathrm{F}(40.3 \%)$ compared to device A $(53.1 \%)$, resulting from a low charge $\eta_{c c}$ in the mixed HJ due to transport losses via recombination in the mixed layer.

One interesting feature is the observed $V_{\mathrm{oc}}$ in the mixed HJ solar cells, which only reaches $0.82 \mathrm{~V}$ compared to $0.9 \mathrm{~V}$ for planar HJ devices. Although the mixed HJ device generates more current, $V_{\text {oc }}$ is lower than the $V_{\text {oc }}$ of the planar HJ device. Such a behavior was also found for the HJ of C60 and $4,4^{\prime}$-bis-( $N, N$-diphenylamino)-quaterphenyl (4P-TPD) using $\quad N, N^{\prime}$-diphenyl- $N, N^{\prime}$-bis $\left(4^{\prime}-[N, N\right.$-bis(naphth-1-yl)amino]-biphenyl-4-yl)-benzidine (Di-NPD) as HTL. ${ }^{18}$ There, it was shown that the $V_{\text {oc }}$ in mixed HJ devices approximately corresponds to the value of $V_{\mathrm{bi}}$, which is given by the difference in the work function of the anode contact and the $p$-doped HTL. In planar HJ the $V_{\text {oc }}$ can exceed the value of $V_{\mathrm{bi}}$ since the planar $\mathrm{HJ}$ has a high diffusive driving force due to the spatial separation of electrons and holes. In mixed HJ, this is not the case because as soon as the voltage significantly exceeds the value of $V_{\mathrm{bi}}$, holes will be injected barrierfree to the interface of the $p$-HTL and the mixed layer, where they recombine with injected electrons from the C60 layer. This recombination current of injected charge carriers compensates the current of photogenerated charge carriers, such that $V_{\mathrm{oc}}$ cannot significantly exceed $V_{\mathrm{bi}}$. When discussing the hybrid PM-HJ device $\mathrm{H}$, it will become evident that it is beneficial to suppress this recombination current by adding a pristine donor layer between $p$-HTL and blend layer.

\section{Hybrid PM-HJ device}

Figure 4(a) compares the $I-V$ characteristics of hybrid PM-HJ device $\mathrm{H}$ with device $\mathrm{A}$ and device $\mathrm{F}$ which show the best results for planar and mixed $\mathrm{HJ}$, respectively. The $V_{\mathrm{oc}}$ of the hybrid PM-HJ device $\mathrm{H}(0.88 \mathrm{~V})$ is increased by approximately $70 \mathrm{mV}$ compared to the result for the mixed $\mathrm{HJ}$ device $\mathrm{E}(0.80 \mathrm{~V}), \mathrm{F}(0.82 \mathrm{~V})$, and $\mathrm{G}(0.81 \mathrm{~V})$. The $V_{\mathrm{oc}}$ of the hybrid PM-HJ device therefore reaches the same values as the planar $\mathrm{HJ}$ devices $\mathrm{A}(0.90 \mathrm{~V}), \mathrm{B}(0.87 \mathrm{~V})$, and $\mathrm{C}$ $(0.89 \mathrm{~V})$. As mentioned above, $V_{\text {oc }}$ in the mixed HJ devices is considered to be limited by $V_{\mathrm{bi}}$, because as soon as the voltage exceeds $V_{\text {bi }}$, injected carriers can easily recombine at the interface between $p$-HTL and mixed layer. By introducing the single layer of DCV6T at this interface, as it is the case in the hybrid PM-HJ device $\mathrm{H}$, a direct recombination of holes on the HTL with electrons from the mixed layer can be suppressed. The result shows that this indeed increases $V_{\text {oc }}$ of the hybrid PM-HJ device to values comparable to planar HJ devices.

The high $j_{\mathrm{sc}}$ of device $\mathrm{H}\left(8.2 \mathrm{~mA} \mathrm{~cm}^{-2}\right.$ at $\left.100 \mathrm{~mW} \mathrm{~cm}^{-2}\right)$ demonstrates a further advantage of the hybrid structure. This value is not reached with the other devices, neither with planar nor with mixed HJ devices. First, the hybrid-PM-HJ device uses high charge separation efficiency. Excitons created in the mixed layer are easily separated because they are always in proximity of a dissociating interface. Excitons created in the adjacent $5.9 \mathrm{~nm}$ single DCV6T layer can as well reach a dissociating interface because this thickness is below the limit of exciton diffusion length. Second, $\eta_{c c}$ of the hybrid PM-HJ device is higher than that of the pure mixed HJ device because it creates charge carriers as efficiently, but simultaneously has lower recombination losses due to the smaller mixed layer thickness. This leads to a better FF of the hybrid PM-HJ device $\mathrm{H}(41.6 \%)$ compared to the mixed HJ devices $\mathrm{G}(38.1 \%)$ and $\mathrm{F}(40.3 \%)$. Nevertheless, the FF of the hybrid PM-HJ device remains low and comparable to results of the mixed $\mathrm{HJ}$ and remains well below the FF of the planar device A $(53.1 \%)$. As can be seen from Fig. 4(b), only for the lowest measured intensity of $2.5 \mathrm{~mW} \mathrm{~cm}^{-2}$ the FF of the hybrid PM-HJ device $\mathrm{H}$ reaches $49 \%$. For increasing intensity, it decreases, while the FF of the planar device A remains at a high level of above $52 \%$. This means that the 
low charge collection efficiency of the mixed layer still plays an important role in the hybrid PM-HJ device that has to be improved to make full use of the hybrid PM-HJ concept.

From the EQE of the hybrid PM-HJ device H, the photocurrent corresponding to the AM 1.5 solar spectrum can be determined by

$$
j_{\mathrm{ph}}=e \int_{\lambda_{1}}^{\lambda_{2}} \Phi_{\text {flux }, \mathrm{AM} 1.5 \mathrm{G}}(\lambda) \operatorname{EQE}(\lambda) d \lambda,
$$

where $e$ is the electron charge and $\Phi_{\text {flux,AM1.5G }}$ is the photon flux of the AM 1.5G solar spectrum. The limits $\lambda_{1}$ and $\lambda_{2}$ were set to 300 and $800 \mathrm{~nm}$ since the EQE is only nonzero within this wavelength range. The calculated photocurrent is $(5.7 \pm 0.4) \mathrm{mA} \mathrm{cm}^{-2}$. By using neutral density filters, the simulator intensity is tuned to match the photocurrent to the calculated value and corresponding values for FF of $43.2 \%$ and $V_{\mathrm{oc}}$ of $0.86 \mathrm{~V}$ are measured. From this, a mismatch corrected power efficiency for the hybrid PM-HJ cell of $\eta_{\mathrm{PCE}}$ $=(2.1 \pm 0.2) \%$ is calculated .

\section{CONCLUSION}

We have studied the performance of the donor material DCV6T in combination with the acceptor material C60 in planar HJ, mixed HJ, and hybrid PM-HJ devices. While $V_{\mathrm{oc}}$ of the planar HJ devices is $0.90 \mathrm{~V}$, mixed HJ devices only exhibit values that are by $70 \mathrm{mV}$ lower. This is attributed to recombination at the interface between HTL and the blend layer. This limits the achievable $V_{\text {oc }}$ to $V_{\mathrm{bi}}$, which is given by the difference in the work functions of anode and HTL. By suppressing this recombination pathway with a pristine DCV6T layer, as is the case for the hybrid PM-HJ device, a higher $V_{\mathrm{oc}}$ of $0.88 \mathrm{~V}$ is achieved.

In the planar HJ device with DCV6T layer thickness of $17.6 \mathrm{~nm}$, signs of an S-shaped $I-V$ characteristic are observed, which show that the transport levels of DCV6T and the used HTL $\alpha$-NPD are not perfectly matched and still exhibit a small energetic barrier. The main advantage of DCV6T is the high IP, which enables high $V_{\text {oc }}$ of up to $0.9 \mathrm{~V}$. Furthermore, the absorption covers the visible spectra from 450 to $680 \mathrm{~nm}$. The investigated devices show that a hybrid PM-HJ structure is the favorable device structure reaching a mismatch corrected power conversion efficiency of
$(2.1 \pm 0.2) \%$. Nevertheless, the hybrid-PM-HJ cell as well as the mixed HJ cell suffers from transport losses in the mixed layer, which limit the FF to a very low value of $41.9 \%$. For future work, it will be important to improve the transport properties of the mixed layer to increase the FF. Furthermore, it is necessary to find a HTL that matches the HOMO of DCV6T in a way that the hole contact is indeed barrierfree, such that the $S$-shaped $I-V$ curve of planar HJ devices can be reduced. This would also result in a $V_{\mathrm{bi}}$ that is better matched to the requirements of the mixed $\mathrm{HJ}$ device.

${ }^{1}$ C. W. Tang, Appl. Phys. Lett. 48, 183 (1986).

${ }^{2}$ S. Uchida, J. G. Xue, B. P. Rand, and S. R. Forrest, Appl. Phys. Lett. 84, 4218 (2004).

${ }^{3}$ D. Gebeyehu, B. Männig, J. Drechsel, K. Leo, and M. Pfeiffer, Sol. Energy Mater. Sol. Cells 79, 81 (2003).

${ }^{4}$ P. Peumans, A. Yakimov, and S. R. Forrest, J. Appl. Phys. 93, 3693 (2003).

${ }^{5}$ N. S. Sariciftci, H. Hoppe, J. Mater. Res. 19, 1924 (2004).

${ }^{6}$ J. G. Xue, B. P. Rand, S. Uchida, and S. R. Forrest, J. Appl. Phys. 98, $124903(2005)$

${ }^{7}$ J. G. Xue, B. P. Rand, S. Uchida, and S. R. Forrest, Adv. Mater. (Weinheim, Ger.) 17, 66 (2005).

${ }^{8}$ M. Yokoyama, M. Hiramoto, and H. Fujiwara, J. Appl. Phys. 72, 3781 (1992).

${ }^{9}$ P. Sullivan, S. Heutz, S. M. Schultes, and T. S. Jones, Appl. Phys. Lett. 84, 1210 (2004).

${ }^{10}$ S. Heutz, P. Sullivan, B. M. Sanderson, S. M. Schultes, and T. S. Jones, Sol. Energy Mater. Sol. Cells 83, 229 (2004).

${ }^{11}$ C. Uhrich, R. Schueppel, A. Petrich, M. Pfeiffer, K. Leo, E. Brier, P. Kilickiran, and P. Bäuerle, Adv. Funct. Mater. 17, 2991 (2007).

${ }^{12}$ R. Schueppel, C. Uhrich, M. Pfeiffer, K. Leo, E. Brier, E. Reinold, and P. Bäuerle, ChemPhysChem 8, 1497 (2007).

${ }^{13}$ K. Schulze, C. Uhrich, R. Schüppel, K. Leo, M. Pfeiffer, P. Bäuerle, E. Brier, and E. Reinold, Adv. Mater. (Weinheim, Ger.) 18, 2872 (2006).

${ }^{14}$ R. Schueppel, K. Schmidt, C. Uhrich, K. Schulze, D. Wynands, J. L. Bredas, E. Brier, E. Reinold, H. B. Bu, P. Bäuerle, B. Männig, M. Pfeiffer, and K. Leo, Phys. Rev. B 77, 085311 (2008).

${ }^{15}$ M. C. Scharber, D. Mühlbacher, M. Koppe, P. Denk, C. Waldauf, A. J. Heeger, and C. J. Brabec, Adv. Mater. (Weinheim, Ger.) 18, 789 (2006).

${ }^{16}$ C. J. Brabec, A. Cravino, D. Meissner, N. S. Sariciftci, T. Fromherz, M. T. Rispens, L. Sanchez, and J. C. Hummelen, Adv. Funct. Mater. 11, 374 (2001).

${ }^{17}$ P. W. M. Blom, L. J. A. Koster, V. D. Mihailetchi, Appl. Phys. Lett. 88, 093511 (2006).

${ }^{18}$ C. Uhrich, D. Wynands, S. Olthof, M. K. Riede, S. Sonntag, K. Leo, B. Männig, and M. Pfeiffer, J. Appl. Phys. 104, 043107 (2008).

${ }^{19}$ S. Yoo, B. Domercq, and B. Kippelen, Appl. Phys. Lett. 85, 5427 (2004).

${ }^{20}$ B. P. Rand, J. Genoe, P. Heremans, and J. Poortmans, Prog. Photovoltaics 15, 659 (2007). 